\title{
Safety and Benefit of Five Times per Week Electroconvulsive Therapy
}

\author{
Kenneth Sakauye ${ }^{1^{*}}$, Farzana Badrun ${ }^{2}$, Andrew Elliott ${ }^{3}$ and William N Yetter $^{4}$ \\ ${ }^{1}$ Department of Psychiatry, University of Tennessee Health Sciences Center, College of Medicine, USA \\ 2 Department of Preventive Medicine, University of Tennessee Health Science Center, USA \\ ${ }^{3}$ University of Tennessee Health Science Center, USA \\ ${ }^{4}$ University of Tennessee Health Sciences Center, College of Medicine, USA
}

*Corresponding author: Kenneth Sakauye, Department of Psychiatry, University of Tennessee Health Sciences Center, College of Medicine, USA, Tel: 9014484572; E-mail: Kenneth.Sakauye2@va.gov

Received Date: September 18, 2014, Accepted Date: October 20, 2014, Published Date: October 27, 2014

Copyright: ( ) 2014, Kenneth Sakauye et al., This is an open-access article distributed under the terms of the Creative Commons Attribution License, which permits unrestricted use, distribution, and reproduction in any medium, provided the original author and source are credited.

\begin{abstract}
Objective: The study aimed to test whether there was more rapid response with daily Electroconvulsive Therapy (ECT) (5 times per week right unilateral) and shorter lengths of stay (LOS) without an increase in adverse effects compared to ECT administered in a routine fashion of 2-3 times/week.
\end{abstract}

Methods: This is a retrospective chart review study of inpatient ECT cases performed over a two year period in a general psychiatric unit. Different practitioners used 5 times/week right unilateral (RUL) ECT, 2-3 times/week right unilateral ECT, or bilateral (BL) ECT. All inpatient records of patients, who received inpatient ECT in a general hospital with 80 psychiatric adult beds, were reviewed in the two year study period. Due to a variation in the quality of documentation, the analysis used proxy variables for improvement and side effects of ECT: LOS following the first ECT to discharge, total treatments in the series, and switches in treatment parameters that indicating poor response or adverse effects.

Results: 78 patients received inpatient ECT. 35 cases were started with RUL ECT in the standard 3 times/week frequency. 32 cases were started with 5 times/week frequency. 11 cases continued BL ECT placement 3 times per week for a repeat ECT series. Diagnosis was not factor in lead placement or frequency of treatment. $90 \%$ of patients had major depression, and $10 \%$ were treated for bipolar disorder or schizophrenia. The number of treatments in a successful series was roughly equivalent irrespective of starting lead placement. 5 times/week RUL lead placement did not lead to significant morbidity based on switch rates. The findings show a trend supporting the use of 5 times/ week RUL ECT as a means to shorten the course of ECT without increasing cognitive morbidity.

Keywords: Electroconvulsive Therapy; Safety; ECT

\section{Background}

Electroconvulsive Therapy (ECT) remains the most effective treatment for depression and many other major psychiatric disorders. Although ECT has long been associated with complaints of memory dysfunction, risk factors for who will develop post-ictal confusion or long-term memory problems have not been definitively identified $[1,2]$, and seem to be transient. Prevention of cognitive side effects has focused mainly on non-dominant hemisphere lead placement $[3,4]$. In naturalistic clinical practice there is a huge variation in lead placement, stimulus parameters, case selection, acceptable health co morbidities, concurrent medications, and frequency of treatments, although the general consensus is to start with right unilateral lead placement, with brief biphasic pulses of square wave stimulations at three to five times threshold $[5,6]$. Measurement of cognitive impairment and depression outcomes are still generally measured by global impression of change. Cognitive change, in particular is generally measured by meta memory assessment since baseline cognitive function before depression, is not verifiable [7]. Bilateral ECT has consistently been associated with more subjective memory complaints than unilateral ECT, including complaints of persistent memory problems even in the absence of objective cognitive deficits on post-ECT testing $[8,9]$.
How often ECT should be administered has been seen as a trade-off between speed of response and minimizing confusion [10]. Three times per week has been the norm, but more or less treatments per week have largely been a matter of clinical judgment. Multiple monitored ECT was often done in the 1980's where several treatments were given in a session. Clinical Procedural Codes (CPT) once included a code for multiple monitored ECTs. Initial comparisons of multiple monitored versus routine treatment showed no difference in effectiveness or safety and had a significantly more rapid course of treatment [11]. However, multiple monitored ECT was subsequently abandoned due to an overall higher rate of cognitive impairment with the dominant mode of bilateral lead placement, and the CPT code has been removed. However, the controversy over the number of ECT per week is still open. The current range of ECT frequency has been five times per week [12] to two to three times per week [10,13] depending upon severity of symptoms. It would be a significant issue for hospitals and insurance if more frequent ECT administration can shorten lengths of stay (LOS) and provide good outcomes without increasing in morbidity. Patients should also find the shorter course of treatments reassuring. 


\section{Methods}

The present study is a retrospective chart review of patterns of ECT administration used by practitioners in an urban general hospital with 80-psychiatric beds. This was a naturalistic outcome study. The data was manually reviewed since electronic medical records were not yet available, and entered into SAS statistical software (SAS Institute, Inc.) for data analysis the decision tree for ECT lead placements and frequency of treatment used by practitioners is listed in Figure 1.

Lead Placement: Initial ECT series utilized right unilateral (RUL) lead placement. Modified right unilateral (Mod RUL) ECT was used if right unilateral seizures seemed focal or of short duration. Bilateral lead placement was used if there was poor response to unilateral treatments or if a prior series required bilateral lead placement for efficacy. If confusion became evident, the frequency of administration was reduced or ECT was discontinued.

Treatment Parameters: The selection of initial frequency of treatments was based on provider preference. One practitioner used RUL 5 times/week routinely while others used 2-3 times/week routinely. Stimulus parameters were empirically determined. Right unilateral ECT generally used 3-5x threshold stimulation and bilateral ECT generally threshold stimulation. ECT was administered with a MECTA Spectrum 5000Q device.

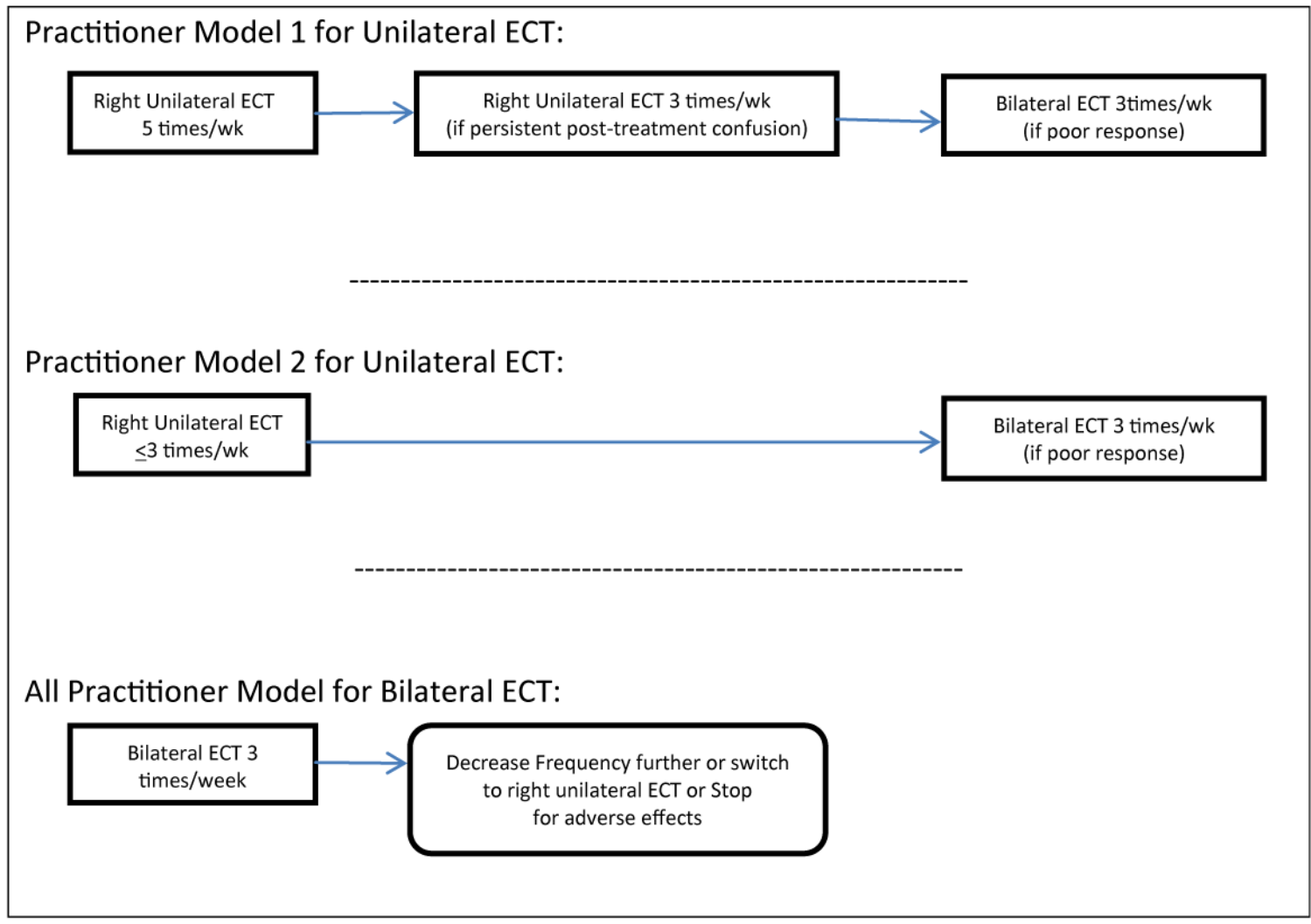

Figure 1: Treatment Decision Tree

Paper-based records were manually reviewed and tallied. Since improvement and adverse effects were rated by global clinical impression and were often implicit, proxy measures were used to provide less subjective measures for improvement and emergent side effects. End of treatment was used as a measure of efficacy. Change in lead placement from unilateral to bilateral was used as a measure of lack of efficacy. Reduction in frequency of administration was used as a measure of clinically significant cognitive complaints. No cases were terminated because of a medical complication.

The following variables were available: demographic information, diagnostic codes, initial lead placement, change in lead placement, number of treatments per week, motor and Electroencephalogram (EEG) based seizure duration, total length of stay, and length of stay following the first ECT.
All inpatient records of patients who received inpatient ECT in a general hospital between July 2009 and July 2011 were reviewed. The hospital records pertaining to ECT and psychiatry were not part of an electronic medical record system so information was manually extracted and entered into SAS Statistical Software (SAS Institute, Inc.).

\section{Results}

35 cases were started with right unilateral ECT in the standard 3 times/week frequency. 32 cases were started with 5 times/week frequency. 11 cases continued bilateral ECT placement 3 times/week for a recurrent ECT series. Diagnosis was not used by the clinicians as a factor in lead placement or frequency of treatment. Most patients had major depression, but $10 \%$ were treated for bipolar disorder or 
Page 3 of 4

schizophrenia. Univariate pair wise comparisons of group outcomes were performed since group sizes and characteristics were not normally distributed (Shapiro-Wilk, W=0.913669, p 0.4899).

There was no significant difference in age, gender, or diagnosis between the 5 times/week unilateral and 3 times/week unilateral groups.

Table 1 shows the average number of treatments and switch rates. The number of switches of RUL placement to Modified RUL or bilateral placement was used as a proxy for lack of efficacy of the original treatment. None of the 3 times/week patients were switched. However six of the 5 times/week patients were switched to either modified right unilateral or bilateral lead placement to improve seizure response or efficacy. The latter was more typical of what was expected because of the lower efficacy rate for unilateral lead placement overall.

\begin{tabular}{|l|l|l|l|l|l|}
\hline $\begin{array}{l}\text { Starting Lead and } \\
\text { frequency }\end{array}$ & $\mathbf{N}(\%)$ & Switch & $\begin{array}{l}\text { N (\%) } \\
\text { Switching }\end{array}$ & Ave Treatments in Series & $\begin{array}{l}\text { LOS in days } \\
\text { (range) }\end{array}$ \\
\hline RUL 2-3 times /wk & $30(39.5 \%)$ & No switches & $29(96.6 \%)$ & $5.75($ SD 3.43) & $12.0(3-44)$ \\
\hline & & Mod RUL & $1(3.33 \%)$ & $4($ SD 0.00) & $24.0(0)$ \\
\hline & & Bilateral & $0(0 \%)$ & 0 & 0 \\
\hline RUL 5x/week & $27(35.5 \%)$ & No switches & $16(59.2 \%)$ & $7.19($ SD 2.4) & $12.66(4-31)$ \\
\hline & & Mod RUL & $3(11.1 \%)$ & $7.0($ SD 3.46) & $9.33(5-16)$ \\
\hline Bilateral ECT 3x/week & $11(14.5 \%)$ & Eventual Bilateral ECT & $6(22.2 \%)$ & $8.83($ SD 2.22) & $22.83(10-46)$ \\
\hline & & No changes & $0(100 \%)$ & $7.45($ SD 4.13) & $22.45(6-88)$ \\
\hline
\end{tabular}

Table 1: Switch Rates, LOS (Length of stay) is from treatment \#1 until discharge, RUL = Right Unilateral lead placement, Mod RUL = Modified Right Unilateral lead placement

The average number of treatments in a series differed unexpectedly for the 3 times/week group. Table 2 shows the 5 times/week RUL group was 7.19 treatments, and bilateral ECT was 7.45 treatments as opposed to 5.75 for the 3times/week RUL group. Usually a RUL ECT series requires more treatments than a course of Bilateral ECT.

The average LOS of the combined right unilateral ECT groups was half the LOS of the bilateral ECT patients.

\begin{tabular}{|l|l|}
\hline Initial Lead Placement & Significance \\
\hline RUL 3x/wk LOS vs. RUL 5x/week LOS & $p=0.4269$ \\
\hline Bilat 3x/wk LOS vs RUL 5x/wk LOS & $p=0.0816$ \\
\hline Bilat 3x/wk LOS vs Mod RUL 3x/wk LOS & $p=0.0090$ \\
\hline Bilat 3x/wk LOS vs Mod RUL 5x/wk LOS & $p=0.0953$ \\
\hline Bilat 3x/wk LOS vs RUL 3x/wk LOS & $p=0.0193$ \\
\hline
\end{tabular}

Table 2: Length of Stay (LOS), RUL=Right Unilateral lead placement, $\sim \mathrm{x} /$ week=times per week, Bilat=Bilateral lead placement

No early termination of bilateral ECT was observed and no reduction of frequency of RUL treatments was observed, suggesting no clinically significant cognitive morbidity in any group.

\section{Discussion}

The findings showed a trend supporting the use of 5 times / week RUL ECT as a means to shorten the course of ECT without increasing cognitive morbidity. The number of treatments in a successful series is roughly equivalent irrespective of starting lead placement, but doing ECT more frequently shortens the time frame. RUL lead placement allows clinically significant improvement without an increase in cognitive impairment (based on switch rates).

In this study, an odd observation was that 3 times/week RUL ECT seemed superior to either 5 times/week ECT or bilateral ECT in terms of fewer number of treatments in a series, no switches to bilateral ECT, or LOS for the course of treatment. This seemed odd in light of purported lower efficacy of RUL ECT compared to BL. This prompted a qualitative chart review of the 2-3 times/week cases. Nonstandardized documentation and lack of detail in the 2-3 times/week cases many charts did not allow any secondary analysis. We concluded that treatment parameters used by the psychiatrists using 2-3 times/ week ECT differed from published American Psychiatric Association (APA) guidelines in terms of inclusion criteria and endpoints for treatment. Our expectation was that the number of treatments in a series would be roughly identical in all groups, and that length of stay would be wholly dependent upon the frequency of treatments.

In looking at $5 \mathrm{x} /$ week RUL ECT group alone, about $20 \%$ of the patients required modification in lead placement to improve efficacy. The modifications were switch to modified right unilateral ECT to improve the quality of seizures, or to bilateral lead placement if Modified RUL lead placement was still suboptimal. The comparison in lengths of stay of 5 times/week RUL cases with Bilateral ECT cases (3 times/week) seemed to be a more useful comparison because of the 2-3 times/week RUL cases seemed so atypical. Of those that completed 5 times/week ECT, the number of treatments was about the same as a BL ECT series, and a trend toward shorter lengths of stay for 5 times/week was seen. No evidence for significant cognitive impairment from 
Citation: Sakauye K, Badrun F, Elliott A, Yetter WN (2014) Safety and Benefit of Five Times per Week Electroconvulsive Therapy . J Psychiatry

Page 4 of 4

treatment was seen, based on further reducing the frequency of ECT throughout the series.

The limitation to this chart review study was the lack of standardized records or scales. Proxy measures for efficacy and adverse effects had to be used because of limitations in the quality of documentation. The quality and reliability of chart data should improve as the Hospital moves toward an electronic Medical Record System. Deviations from practice guidelines by some psychiatrists were an inadvertent finding that is being corrected by the Hospital.

In conclusion, 5 times/week RUL ECT seems to be well tolerated and can shorten the length of a series of ECT.

\section{References}

1. Kikuchi A, Yasui Furukori N, Fujii A, Katagai H, Kaneko S (2009) Identification of predictors of post-ictal delirium after electroconvulsive therapy. Psychiatry and Clinical Neurosciences, 63: 180-185.

2. Hausner L, Sartorius DM, Frolich L (2011) Efficacy and Cognitive Side Effects of Electroconvulsive therapy (ECT) in depressed elderly inpatients with coexisting mild cognitive impairment of dementia. Journal of Clinical Psychiatry 72: 97-7.

3. Abrams R (2007) Does Bilateral ECT Cause Persistent Cognitive Impairment. Journal of ECT. 23: 61-62.

4. Crowley K, Pickle J, Dale R, Fattal O (2008) A Critical Examination of Bifrontal Electroconvulsive Therapy: Clinical Efficacy, Cognitive Side Effects, and Directors for Future Research. Journal of ECT 24: 268-271.

5. Sackeim HA, Prudic J, Devanand DP, Nobler MS, Lisanby SH, et al. (2000) A Prospective, Randomized, Double-blind Comparison of
Bilateral and Right Unilateral Electroconvulsive Therapy at Different Stimulus Intensities. Archives of General Psychiatry 57: 425-434.

6. Weiner RD, Fink M, Hammersley DW, Small IF, Moench LA, et al. (1990) The American Psychiatric Association Task Force on Electroconvulsive Therapy, The Practice of Electroconvulsive Therapy: Recommendations for Treatment, Training, and Privileging, American Psychiatric Association, Washington DC

7. Brakemeier EL, Berman R, Prudic J, Zwillenberg K, Sackheim HA (2008) Self-evaluation of the cognitive effects of electroconvulsive therapy. Brain Stimulation, 1: 16-26.

8. Squire LR, Chance PM (1975) Memory functions six to nine months after electroconvulsive therapy. Archives of General Psychiatry 32: 1557-64.

9. Prudic J, Peyser S, Sackeim HA (2000) Subjective Memory Complaints: A Review of Patient Self-Assessment of Memory after Electroconvulsive Therapy. The Journal of ECT 16:121-132.

10. Shapira B, Tubi N, Lerer B (2000) Balancing Speed of Response to ECT in Major Depression and Adverse Cognitive Effects: Role of Treatment Schedule. The Journal of ECT 16:109.

11. Berens ES, Yesavage JA, Leirer VO (1982) A Comparison of Multiple and single electroconvulsive therapy. Journal of Clinical Psychiatry 43: 126-8. 1.

12. Hirose S, Ashby CR, Mills MJ (2001) Effectiveness of ECT Combined with Risperidone Against Aggression in Schizophrenia. The Journal of ECT 17: 22-26.

13. Charlson F, Siskind D, Doi SAR, Mc Callum E, Broome A, et al. (2011) ECT efficacy and treatment course: A systematic review and metaanalysis of twice vs thrice weekly schedules. Journal of Affective Disorders. 